\title{
"Desarrollo de un Modelo Dinámico para determinar la incidencia de los factores contaminantes del aire en la población de Lima Metropolitana"
}

Mg. Juan Manuel Rivera Poma ${ }^{1}$

\begin{abstract}
RESUMEN
La atmosfera es un elemento principal para la conservación de la calidad de vida que deseamos tener. El crecimiento de la población es uno de los principales elementos que influye en la contaminación atmosférica. Por lo que se considera necesario analizar las relaciones de la contaminación y sus efectos en la población. El efecto de estos parámetros en la contaminación y salud de la población ha sido estudiado como una relación de causa-efecto planteándose un modelo dinámico de manera que usando un programa de simulación computarizada, podemos conocer su comportamiento en el futuro. De esta manera estaríamos en las posibilidades de reducir la contaminación del aire, o al menos evitar su incremento, mediante el control sobre estos parámetros.
\end{abstract}

Palabras claves: Simulación atmosférica, Contaminación atmosférica, Factores contaminantes, Control de contaminación atmosférica, contaminación - población.

DEVELOPMENT OF A DYNAMIC MODEL TO DETERMINE THE IMPACT OF AIR POLLUTANT FACTORS IN THE POPULATION OF LIMA

\section{ABSTRACT}

The atmosphere is a major element in the preservation of the quality of life we want. The population growth is one of the main factors that influence air pollution, for what is considered necessary to analyze the relationship of pollution and its effects on the population. The effect of these parameters on pollution and population health has been studied as a cause and effect so that using a computer simulation program; we can study their behavior in the future. In this way we would be in the potential for reducing air pollution, or at least prevent its increase, by control of these parameters.

Keywords: atmospheric simulation, air pollution, pollution factors, air pollution control, pollution population

\section{INTRODUCCIÓN}

La mala calidad del aire existente en la ciudad de Lima metropolitana ${ }^{2}$, que no permiten a sus habitantes gozar de un aire limpio y una mejor salud, exige un mejor conocimiento de los factores que afectan y contaminan el aire.

Siendo la principal fuente de contaminación del aire en la ciudad de Lima las unidades de transporte, que producen grandes cantidades monóxido de carbono, óxidos de nitrógeno y compuestos orgánicos volátiles; Los camiones y buses a motor Diesel del servicio de transporte público masivo son los mayores agentes contaminantes por la cantidad de óxidos de nitrógeno y material particulado que emiten.

La población de Lima y Callao ha crecido desde 0.91 millones en 1940 a 9.3 millones al 2010, constituyendo el $33 \%$ de la población nacional, según cifras de INEI. ${ }^{3}$

Su crecimiento, en gran parte de la ciudad, ha sido en forma desordenada en aspectos urbanos e industriales, sin planificación establecida; en muchos casos se establecieron por invasiones de la población, careciendo de agua y desagüe.

Sus pobladores en su mayoría son integrados por inmigrantes de provincias con baja cultura de saneamiento y limpieza.

La situación actual de la ciudad de Lima, considerada una de las más contaminadas del hemisferio exige un llamado de atención por parte del gobierno central, municipalidades e instituciones responsables de su cuidado y control.

El modelo propuesto permitirá reconocer los factores contaminantes que afectan a la población y proponer políticas públicas que permitan obtener un aire limpio. Evitando la propagación de enfermedades y desarrollo de focos infecciosos.

\section{PRELIMINARES}

El aire tiene la función, entre otras, la de transmitir el sonido, filtrar y amortiguar los rayos del sol, dispersar la luz y mantener el equilibrio térmico. La energía solar ayuda en la purificación del

Juan Manuel Rivera Poma, Ingeniero Industrial, Magister en Ingeniería Industrial con mención en Producción de la U.N.M.S.M., Docente universitario en la U.N.M.S.M. y en la Universidad de Ingeniería y Tecnología - UTEC, jmriverap@yahoo.es

2 Lima Metropolitana incluye las ciudades de Lima y del Callao

3 INEI: Instituto Nacional de Estadística e Informática - Perú 
aire atmosférico; las plantas a través del proceso de la fotosíntesis absorben el bióxido de carbono y desprenden el oxígeno purificando el aire.

La actividad industrial, emite contaminantes en forma permanente, tales como anhídrido sulfúrico, compuestos de azufre, óxidos de nitrógeno, fenoles, etc., así como la disposición final de residuos sólidos emiten óxidos de azufre y nitrógeno, anhídridos y ácidos orgánicos.

El parque automotor formado por vehículos obsoletos de transporte personal y público, emiten gases contaminantes que afectan la salud del poblador limeño.

Las fuentes antropogénicas incluyen la quema de combustibles sólidos como la madera y el carbón, las actividades agrícolas como la fertilización y almacenamiento de granos y la industria de la construcción.

El presente trabajo de investigación es de tipo cualitativo. Las fuentes de información que se emplearán son:

Fuentes de datos primeros. Registro de datos de DIGESA 4

Fuente de datos secundarios INEI ${ }^{5}, \mathrm{MINSA}^{6}, \mathrm{MEM}^{7}$.

\section{MODELO DINÁMICO DE CONTAMINACIÓN ATMOSFÉRICA}

\section{Consideraciones}

La protección del Medio Ambiente tiene un mayor significado en nuestras vidas cada día, por lo que es necesario otorgarle mayor importancia a la conservación y cuidado de nuestro planeta y en especial a las ciudades urbanas grandes.

Si aplicamos oportunamente métodos sistémicos, como una nueva ciencia en el planteamiento de métodos apropiados en resolver los diferentes factores que intervienen en la contaminación, podremos resolver con mayor éxito muchos problemas. Por lo que la ejecución de un programa depende del conocimiento y familiaridad del medio ambiente y sus contaminantes.

En la Zona Metropolitana de Lima, y ciudades que tiene particulares condiciones climáticas, podemos relacionar la contaminación atmosférica con la salud y el crecimiento de la población usando un sistema dinámico.
Para su estudio se ha desarrollado Diagramas Causales, considerando los contaminantes mas representativos y de mayor incidencia en la población. Estos diagramas casuales fueron transferidos a diagramas de flujos y finalmente se realizó una simulación utilizando el software STELLA, considerando estadísticas y tasas reales.

\section{TIPOS DE CONTAMINANTES}

Son 5 los tipos de materiales que considerados como los mayores contaminantes del aire y constituyen más el $90 \%$ de los factores de contaminación del aire en las ciudades, estas son:

- Monóxido de carbono (CO)

- Oxido nitrógeno (NOx)

- Hidrocarburo (HC)

- Oxido de azufre (SOx)

- Sólidos suspendidos (PM-10, PM-2.5)

\section{MODELAMIENTO DE LA CONTAMINACIÓN DEL AIRE}

\section{Declaración del Problema}

La situación de Lima y Callao, es la que refleja a una ciudad que ha crecido en forma desordenada y sin control, mezclándose los aspectos urbanos e industriales sin una planificación establecida.

El crecimiento del parque automotor con bastante antigüedad y la adición de una gran flota de vehículos de segundo uso utilizado como taxis; el crecimiento de asentamientos humanos con personas que en su mayoría son inmigrantes que viven sin las condiciones apropiadas de agua y desagüe o condiciones mínimas de higiene; la falta de educación y conciencia de preservar el medio ambiente en aspectos de salubridad, uso y mantenimiento de equipos; la falta en el cumplimiento de las leyes y reglamentos, entre otras, hace necesario el estudio de los factores de la contaminación, con criterio técnico.

Considerando que la contaminación del aire tiene efectos en la población y que su comportamiento depende de múltiples variables de características naturales, geológicas y humanas se ha efectuado un análisis de comportamiento dinámico.

En el desarrollo del modelo relaciona la contaminación del aire, la población y la salud pública. Esta-

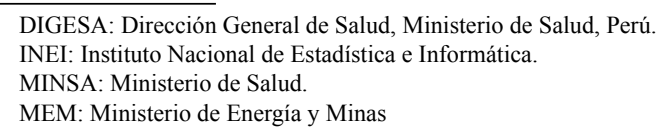


"Desarrollo de un Modelo Dinámico para determinar la incidencia de los factores contaminantes del aire en la población de Lima MetroPOLITANA"

bleciendo variables que influyen en la contaminación del aire de la ciudad en condiciones reales.

\section{Variables del modelo}

Las principales variables del modelo son:

1. Índice de contaminación del aire.

El Índice de contaminación dependerá de la siguiente variable:

2. Concentración del contaminante atmosférico (PM-10), en $\mathrm{mg} / \mathrm{m} 3$.

Se calcula la concentración anual del PM-10 con los valores de las siguientes variables:

3. Tasa de concentración del PM-10.

4. Cantidad de PM-10 en la atmósfera, en Tn/año.

5. Total emisión automotriz, industrial y doméstica, en Tn/año.

6. Volumen de aire en Lima Metropolitana y el Callao, en m3.

La concentración anual del PM-10 se obtiene de la relación entre la cantidad de PM-10 en la atmósfera y el volumen de aire en Lima Metropolitana. La Cantidad de PM-10 en la atmósfera se resulta multiplicando la tasa de concentración del PM-10 y la variable total emisión automotriz, industrial y doméstica. Esta variable es la suma de las siguientes tres variables:

7. Emisión de contaminantes del parque automotriz, en tn/año.

8. Emisión de contaminantes del sector industrial, en tn/año.

9. Emisión de la población o doméstica, en tn/año.

Cada una de estas variables es afectada por otras tres variables:

10. Tasa de emisión del parque automotriz.

11. Tasa de emisión del sector industrial.

12. Tasa de emisión de la población.

Hay muchas variables que deberían ser listados adicionalmente; sin embargo, la que más cubre es la variable:

\section{Población de la ciudad de Lima Metropolitana.}

La población crece en cantidad con los nacimientos y los inmigrantes y decrece con las muertes por enfermedades producto de la contaminación, y las muertes por causas diferentes. Las actividades que realiza la población también ayudan a la emisión de contaminantes atmosféricos como es el caso de actividades comerciales, restaurantes (chimeneas de cocinas), quema de residuos sólidos (basura).

La variable: tasa de emisión de la población ( $\left.N^{\circ} 12\right)$, indica la emisión de contaminantes al año en toneladas por cada 10,000 habitantes. Con el número de habitantes al año se puede obtener la emisión de la población o doméstica, en toneladas al año.

La población de la ciudad afecta otras 5 variables:

14. Tasa de nacimientos.

15. Tasa de muerte por contaminación.

16. Tasa de muerte por otras enfermedades.

17. Tasa de inmigrantes.

18. Tasa de emigrantes.

El aumento de la contaminación del aire pone en peligro la Salud Pública, de manera que las siguientes variables pueden ser influenciadas:

19. La tasa de infección con enfermedades debido a la contaminación del aire.

20. Número de infectados.

21. Población enferma.

22. Número de personas curadas.

23. Tasa de personas curadas.

24. Número de muertes por causa de la contaminación.

25. Tasa de muertes por la contaminación.

\section{Hipótesis Dinámica}

Determinar las relaciones de la contaminación del aire y sus efectos en la salud pública, proyectándolas a un periodo de 10 años, determinado sus condiciones futuras.

\section{Diagrama de Modelos Causales}

En este diagrama se interrelaciona las variables de contaminación, la salud pública y la población, que según su incidencia pueden ser afectados en el modelo.

\section{Diagrama Causal (Ver Gráfico1)}

En el proceso de desarrollo del modelo, después de determinar la influencia de las variables, mostramos las relaciones causales entre dos o más variables y sus efectos sobre la otra. Trazando lazos para mostrar la relación entre las variables, indicamos que relación es positiva y que otra es negativa. El lazo positivo nos indicara un crecimiento exponencial mientras que los lazos negativos sugieren un balance y/o disminución en el uso o crecimiento. 
Gráfico 1: Diagrama Causal del Modelo Dinámico

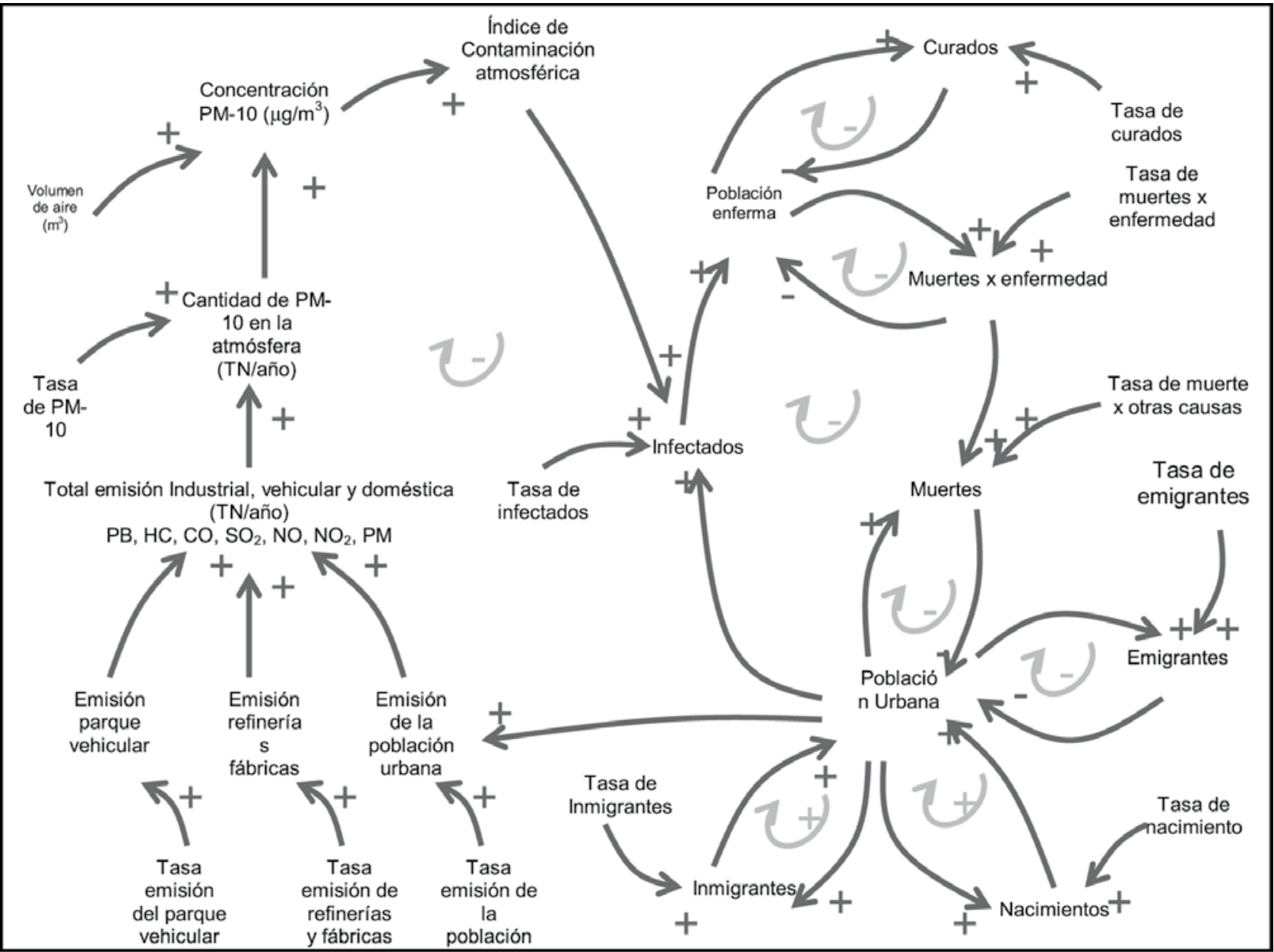

Fuente: Elaborado por el propio Investigador JMRP

\section{Desarrollo del Modelo Dinámico de Contaminación}

El Modelo relaciona la contaminación del aire con la salud de la población, su crecimiento, decrecimiento, las enfermedades contaminantes y muertes con sus respectivas tasas de crecimiento para un periodo de 10 años, luego son proyectados para un periodo adicional de 10 años.

En este modelo se ha determinado 8 circuitos casuales: de los cuales 2 son positivos y 6 negativos. (Ver cuadro I)

\section{Descripción del modelo:}

1. En el primer circuito hay un lazo generalmente negativo entre el índice de contaminación del aire y el número de personas infectadas por la contaminación, la población urbana, emisión de la población, total emisión Industrial, vehicular y doméstica, cantidad de PM-10 en la atmósfera y la concentración del PM-10.

2. En el segundo circuito existe un lazo negativo entre el la población enferma y el número de muertes por contaminación, el total de muertes que incluyen a muertes por otras causas, la población urbana y el número de infectados.

3. En el tercer circuito hay otro lazo negativo entre la población urbana y el número de emigrantes por año. Un incremento de número de emigrantes al año hará que la población urbana disminuya.

4. En el cuarto circuito, también tenemos otro lazo negativo entre la población urbana y el número de muertes por año, que representa al número de muertes por la contaminación y en número de muertes por otras causas.

5. En el quinto, tenemos un lazo positivo entre la población urbana y el número de nacimientos al año. El número de nacimientos al año crecerá conforme se incremente la población urbana. La población crecerá si el número de muertes y emigrantes al año es menor al número de nacimientos e inmigrantes. 
6. En el sexto circuito, tenemos otro lazo positivo entre la población urbana y el número de inmigrantes. La población urbana crecerá conforme aumente en número de inmigrantes.

7. En el séptimo, tenemos un lazo negativo entre la población enferma y el número de personas curadas de las enfermedades por contaminación ambiental. Si aumenta el número de personas curadas la población enferma disminuye.

8. El último circuito se tiene un lazo negativo entre la población enferma y el número de muertes al año por causa de la contaminación atmosférica. Un incremento del número de muertes por esta causa hará que disminuya la población enferma.

El modelo se desarrolla dinámicamente en una interacción permanente entre estos ocho lazos. Los flujos de cada una de las variables y explicaciones del desarrollo de cada caso han sido interrelacionados con el programa Stella 9.0, obteniéndose la simulación de su comportamiento para los próximos 10 años (ver gráfico 2).

Gráfico 2: "simulación del modelo dinámico de contaminación"

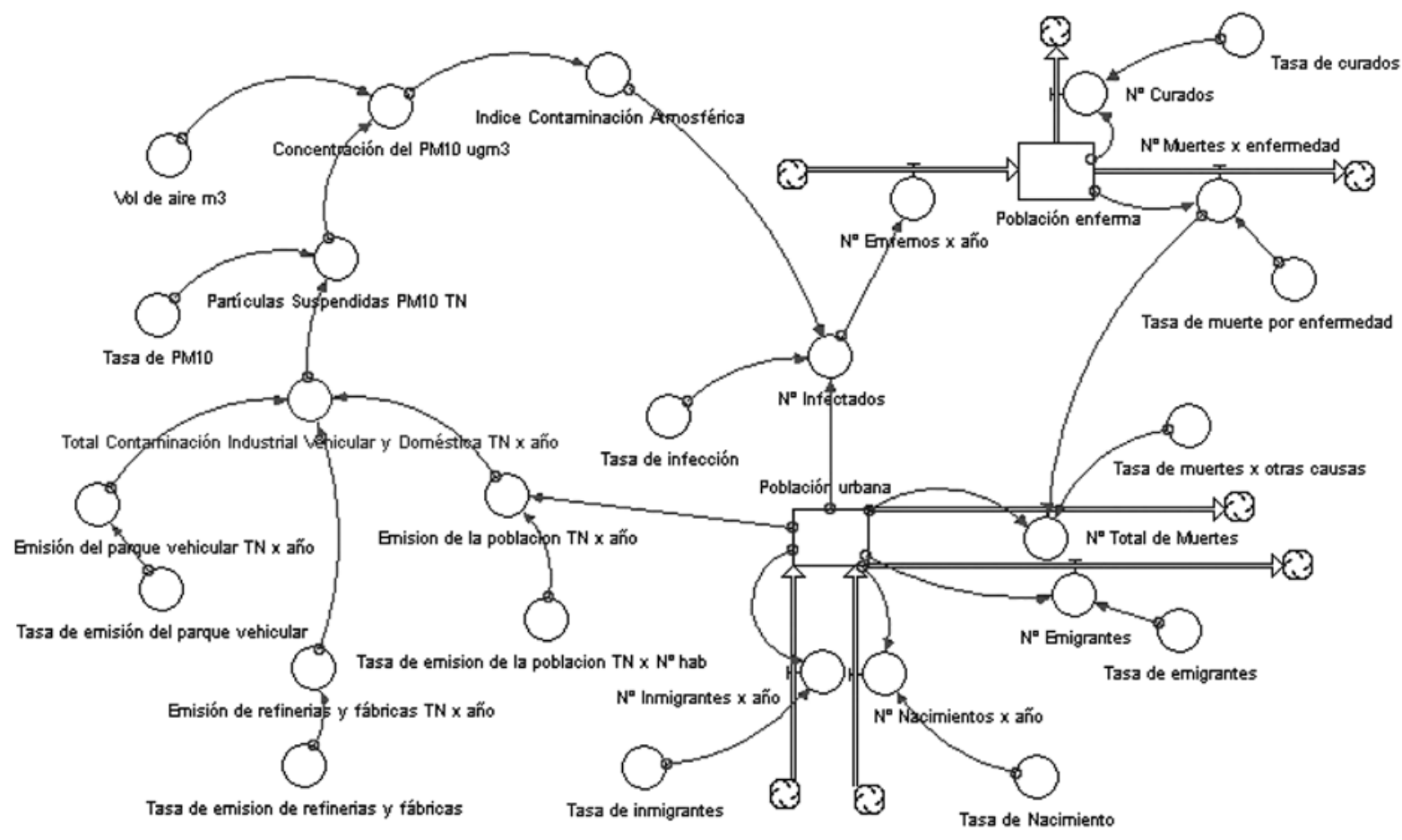

Fuente: Elaborado por el propio Investigador JMRP

\section{Diagrama de Flujo del Modelo Dinámico}

El diagrama de flujo visualiza las variables del modelo de manera que los procesos son direccionados con la información y características consideradas, integrando la estructura principal y la base física del modelo. El tamaño de población (en cantidad de personas), la cantidad de contaminantes en la atmósfera (en toneladas), la concentración de los contaminantes PM-10 (en $\mathrm{mg} / \mathrm{m} 3$ ), etc. Son determinados en los diagramas como una cantidad o una fórmula numérica y lógica. En los diagramas de flujo del modelo de la contaminación atmosférica, los parámetros y variables son divisibles en siguientes categorías: a) Variable de nivel: población urbana y población enferma.

b) Variable de tasa: número de nacimientos por año, número de inmigrantes por año, número de muertes por año, número de emigrantes por año, número de muertes por enfermedad por año, número de personas curadas por año, número de enfermos por año.

c) Variable auxiliar: emisión del parque vehicular, emisión de refinerías y fábricas, emisión de la población, total contaminación, industrial vehicular y doméstica (los sólidos suspendidos: polvo, $\mathrm{PB}, \mathrm{HC}, \mathrm{CO}, \mathrm{SO} 2, \mathrm{NO} 2, \mathrm{Y} \mathrm{NO}$ ), partículas 
suspendidas PM-10, concentración del PM-10, índice de contaminación atmosférica, número de infectados.

d) Constantes: tasa de nacimientos, tasa de inmigrantes, tasa de emigrantes, tasa de muerte por enfermedad de contaminantes, tasa de muerte por otras causas, tasa de curados, tasa de infección, tasa de emisión del parque vehicular, tasa de emisión de refinerías y fábricas, tasa de emisión de la población, tasa de PM10 , volumen de aire.

e) Tabla de funciones: después de obtener el diagrama de flujo, ingresamos los números y fórmulas para cada uno de nuestros niveles de tasa y variables auxiliares así como las constantes y la tabla de funciones. Ahora se tiene un modelo completo. Luego procedemos a correr el programa, obteniendo como resultado la evolución de los contaminantes por un periodo de 10 años (ver tablas 1, 2 y 3). En estas tablas se muestra los diversos comportamientos de la emisión del parque vehicular, industrial (refinerías) y de la población; así como los totales de contaminación existente en la atmosfera de la ciudad en toneladas, también se muestra el total de partículas suspendidas y su incremento en los diferentes años. Estos resultados muestran una situación futura preocupante.

Gráfico 3: Simulación del Modelo Dinámico de Contaminación

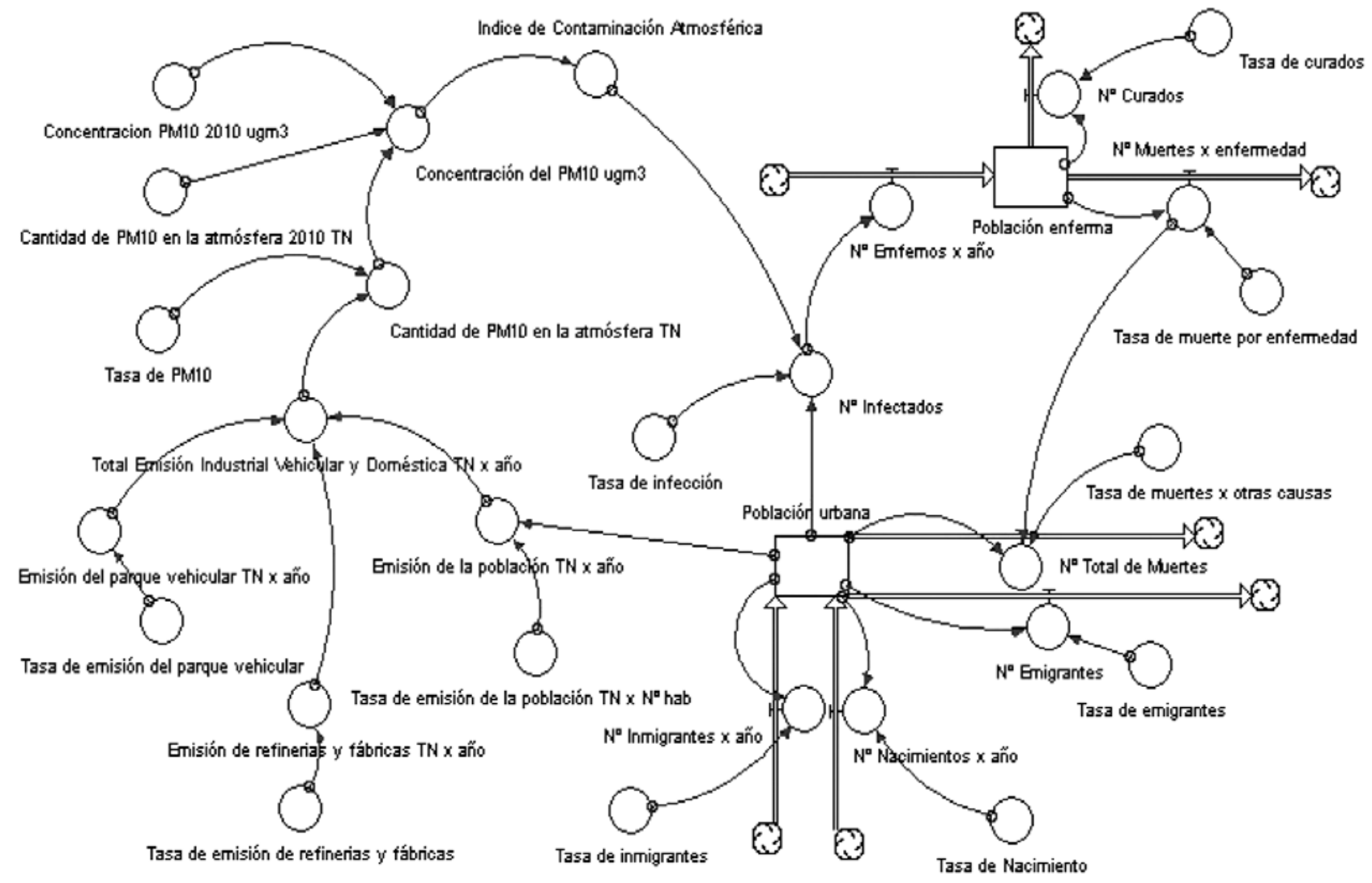

Fuente: Elaborado por el propio Investigador JMRP 
"Desarrollo de un Modelo Dinámico para determinar la incidencia de los factores contaminantes del aire en la población de Lima MetroPOLITANA"

Tabla1: Evolución de la población urbana, inmigrantes, nacimientos, muertes y Emigrantes

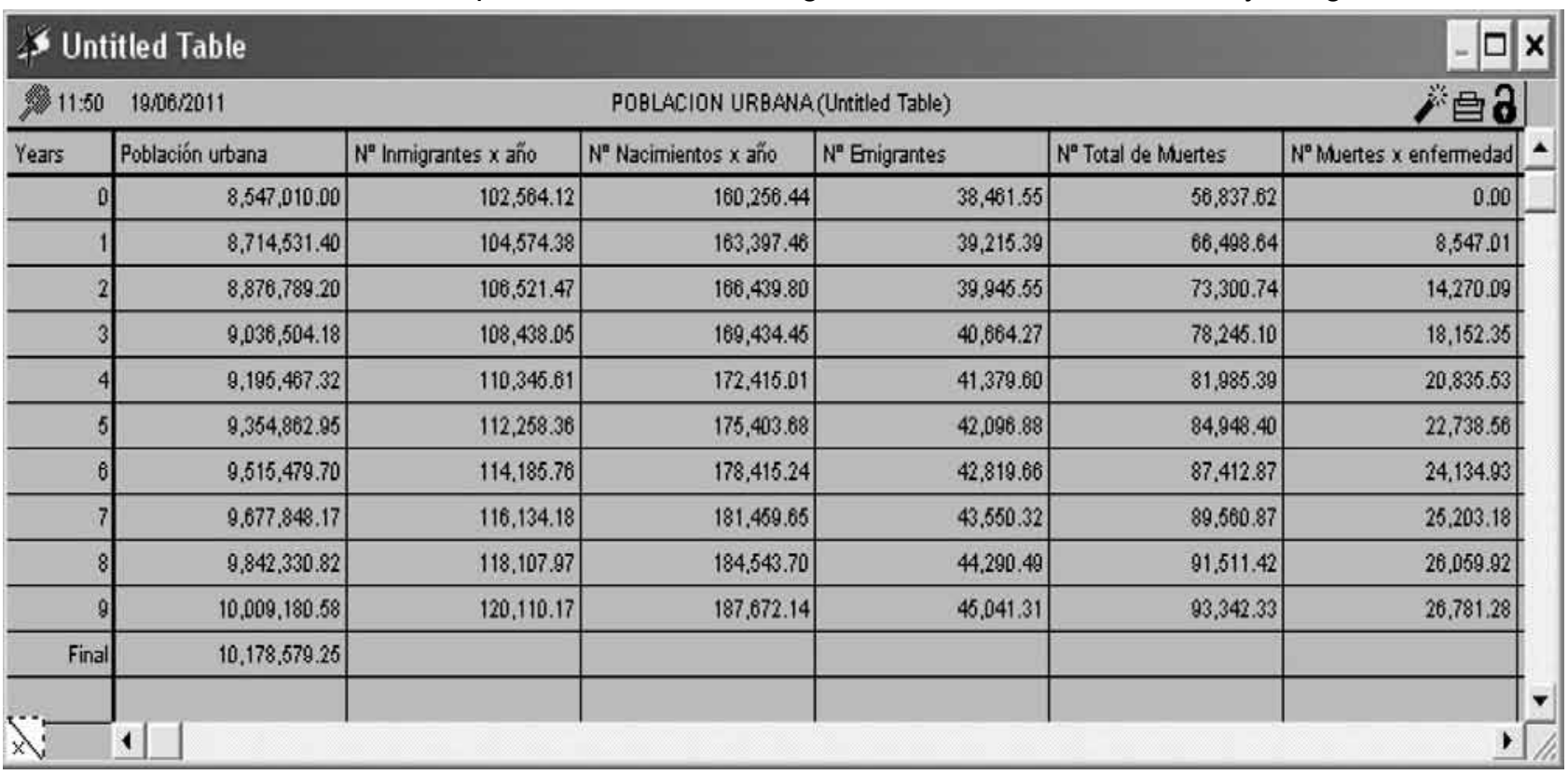

Fuente: Elaborado por el propio Investigador JMRP

Tabla 2: EVOLUCIÓN DE CONTAMINANTES ATMOSFÉRICOS, CONCENTRACIÓN DEL PM-10 Y EL INDICADOR DE CONTAMINACIÓN Considerando la disminución de la contaminación industrial (5\%) y Vehicular $(10 \%)$ anual

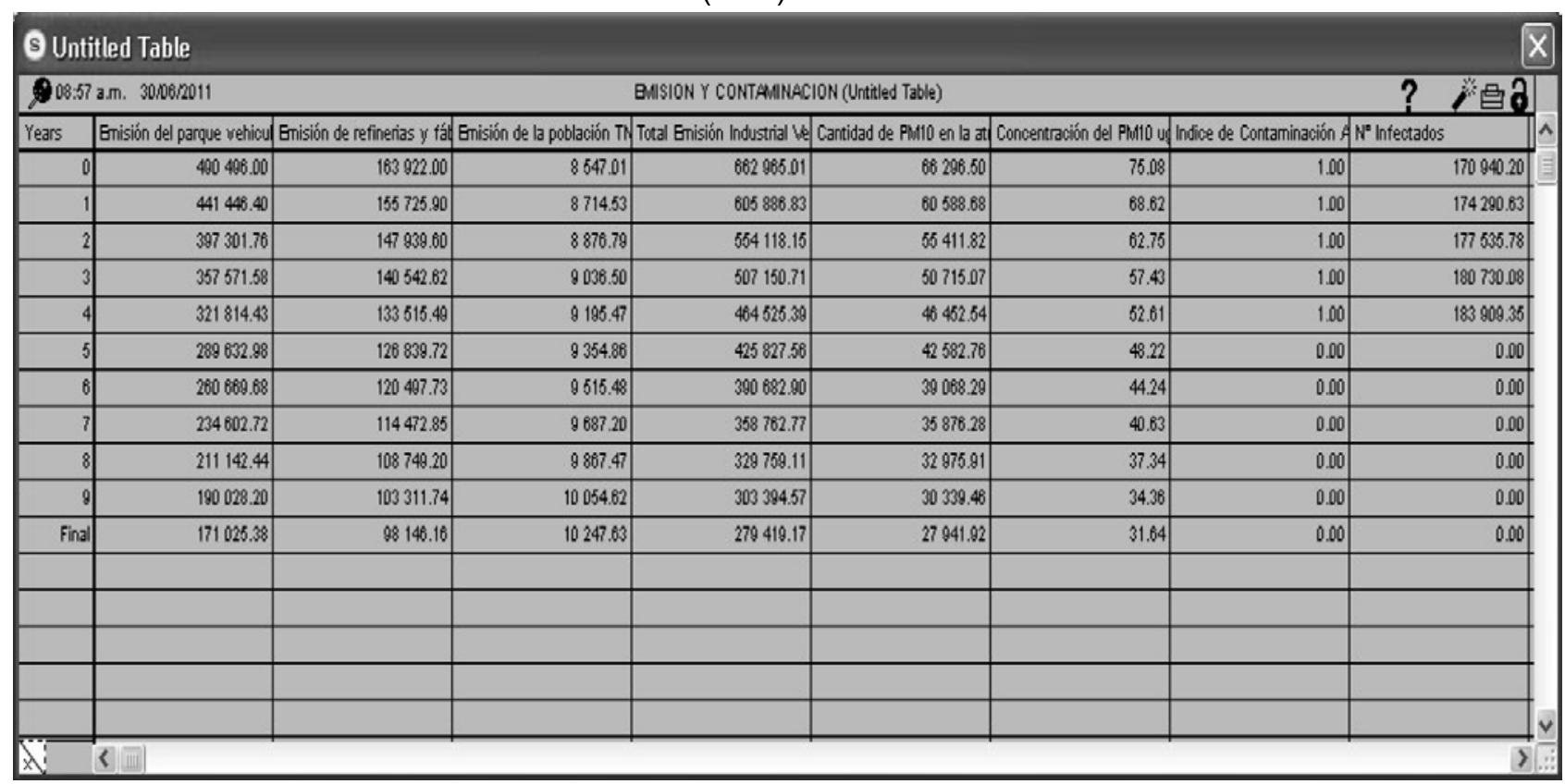

Fuente: Elaborado por el propio Investigador JMRP

f) En la tabla 3, se muestra el crecimiento de la población, estimándose 11'894,000 habitantes para el año 2,021. Así como los inmigrantes, nacimientos, muertes y emigrantes.

g) En la tabla 3, se estima la población enferma, muertes y curados, para el mismo periodo. h) Teniendo en cuenta las mismas condiciones establecidas, se simularon diversos escenarios de mejora, siendo una de la más probable de solución, la disminución de $5 \%$ en la contaminación industrial y un $10 \%$ en la vehicular. Lográndose reducir la contaminación y sus efectos en un periodo de 10 años (ver cuadro VII). 
Tabla 3: Evolución De La Población Urbana, Inmigrantes, Nacimientos, Muertes Y Emigrantes

\begin{tabular}{|c|c|c|c|c|c|c|c|}
\hline \multicolumn{6}{|c|}{ \& Untitled Table } & \multirow{2}{*}{ 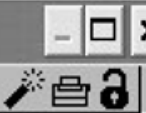 } & \multirow[t]{3}{*}{$x$} \\
\hline \multirow{2}{*}{$\frac{123: 15}{\text { Years }}$} & \multicolumn{2}{|l|}{$24 / 04 / 2011$} & \multicolumn{3}{|c|}{ Poblacion urbana (Untitled Table) } & & \\
\hline & Población urbana & $\mathrm{N}^{0}$ Inmigrantes $\mathrm{x}$ año & $N^{0}$ Nacimientos $x$ an̂́o & $\mathrm{N}^{0}$ Total de Muertes & $N^{0}$ Enigrantes & & \\
\hline 0 & $8,547,010.00$ & $42,735.05$ & $427,350.50$ & 85.470 .10 & $85,470.10$ & & \\
\hline 1 & $8,846,155.35$ & $44,230.78$ & $442,307.77$ & 92.735 .06 & 88.461 .55 & & \\
\hline 2 & $9,151,497.28$ & $45,757.49$ & 457.574 .86 & $99,570.53$ & $91,514.97$ & & \\
\hline 3 & $9,463,744.13$ & $47,318.72$ & 473.187 .21 & $106,060.41$ & $94,637.44$ & & \\
\hline 4 & $9.783,552.20$ & $48,917.76$ & 489.177 .61 & $112,276.92$ & $97,835.52$ & & \\
\hline 5 & $10,111,535.13$ & $50,557.68$ & $505,576.76$ & 118.282 .32 & 101.115 .35 & & \\
\hline 6 & $10,448,271.90$ & $52,241.36$ & $522,413.59$ & $124,130.41$ & $104,482.72$ & & \\
\hline 7 & $10,794,313.73$ & $53,971.57$ & 539.715 .69 & $129,867.81$ & $107,943.14$ & & \\
\hline 8 & $11,150,190.04$ & $55,750.95$ & $557,509.50$ & $135,535.03$ & $111,501.90$ & & \\
\hline 9 & $11,516,413.56$ & 57.582 .07 & $575,820.68$ & $141,167.39$ & $115,164.14$ & & \\
\hline Final & $11,893,484.78$ & & & & & & \\
\hline & & & & & & & \\
\hline & & & & & & & \\
\hline & & & & & & & \\
\hline & & & & & & & \\
\hline & & & & & & & \\
\hline 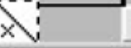 & 4 & & & & & - & \\
\hline
\end{tabular}

Fuente: Elaborado por el propio Investigador JMRP

Tabla 4: Evolución de La Población: Urbana, Enferma, Curada, Y Muertes

\begin{tabular}{|c|c|c|c|c|c|c|}
\hline \multicolumn{5}{|c|}{$\$$ Untitled Table } & \multirow{2}{*}{\multicolumn{2}{|c|}{$\begin{array}{l}-\square \times \\
\text { 早 }\end{array}$}} \\
\hline $823: 25$ & $24 / 04 / 2011$ & \multicolumn{3}{|c|}{ Poblacion enferma (Untitled Table) } & & \\
\hline Years & Población urbana & Población enferma & $N^{2}$ Muertes $x$ enfermedad & $\mathrm{N}^{0}$ Curados & $N^{0}$ Total de Muertes & $\Delta$ \\
\hline 0 & $8,547,010.00$ & 0.00 & 0.00 & 0.00 & $85,470.10$ & \\
\hline 1 & $8,846,155.35$ & $85,470.10$ & $4,273.51$ & $8,547.01$ & $92,735.06$ & \\
\hline 2 & $9,151,497.28$ & $161,111.14$ & $8,055.56$ & 16.111 .11 & $99,570.53$ & \\
\hline 3 & $9.463,744.13$ & 228.459 .44 & $11,422.97$ & $22,845.94$ & $106,060.41$ & \\
\hline 4 & $9.783,552.20$ & 288.827 .97 & $14,441.40$ & $28,882.80$ & $112,276.92$ & \\
\hline 5 & $10,111,535.13$ & 343.339 .29 & $17,166.96$ & $34,333.93$ & $118,282.32$ & \\
\hline 6 & $10,448,271.90$ & $392,953.75$ & $19,647.69$ & $39,295.38$ & $124,130.41$ & \\
\hline 7 & $10,794,313.73$ & 438.493 .41 & $21,924.67$ & $43,849.34$ & $129,867.81$ & \\
\hline 8 & $11,150,190.04$ & $480,662.53$ & $24,033.13$ & $48,066.25$ & $135,535.03$ & \\
\hline 9 & $11,516,413.56$ & $520,065.05$ & $26,003.25$ & $52,006.51$ & $141,167.39$ & \\
\hline Final & $11,893,484.78$ & $557,219.43$ & & & & \\
\hline & & & & & & \\
\hline & & & & & & \\
\hline & & & & & & \\
\hline 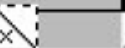 & 1 & & & & & \\
\hline
\end{tabular}

Fuente: Elaborado por el propio Investigador JMRP 
"Desarrollo de un Modelo Dinámico para determinar la incidencia de los factores contaminantes del aire en la población de Lima MetroPOLITANA"

\section{CONCLUSIONES}

Mediante el desarrollo del Modelo Dinámico propuesto en el Diagrama Causal mostrado en el grafico 1 y aplicada al software de Estella 9.0, se obtuvieron las siguientes conclusiones:

1. Se realiza los análisis estadísticos de los principales contaminantes durante un periodo de 10 años; determinándose que los contaminantes de mayor peligro son las partículas en suspensión PM-10 y PM-2.5.

2. Mediante la aplicación de modelos dinámicos se analizaron las relaciones de los contaminantes con la salud pública, para luego ser proyectada para el periodo 2011 al 2021, obteniéndose un comportamiento creciente, mostrando el serio peligro de la salud en los habitantes.

3. En el modelo desarrollado se analizaron diversos escenarios de comportamiento de la contaminación. Obteniéndose que mediante una reducción del $10 \%$ anual de la contaminación vehicular y del $5 \%$ anual de la contaminación industrial permitiría, que en un periodo razonable de 10 años, se pueda obtener una atmosfera limpia en la ciudad.

4. Las principales fuentes de contaminación en la ciudad son: las Industriales, las vehiculares y las domesticas, siendo las vehiculares las de mayor proporción $(70 \%)$

5. Como principal conclusión se determinó la posibilidad de reducción de la contaminación mediante el control estricto de los focos contaminantes en la ciudad, para lo cual, se propone la implementación de políticas públicas y el control de su aplicación por las áreas competentes tales como: Municipalidades, Gobiernos Regionales y Gobierno Central. Estas políticas deberán enfatizar en su aplicación y cumplimiento.

\section{RECOMENDACIONES}

Las principales acciones propuestas a realizar por las entidades mencionadas son:

1. Limpieza de los contaminantes atmosféricos de todas las calles de la ciudad, recogiendo la polución y depositándola en lugares apropiados que eviten su contaminación. (uso de aspiradoras en forma intensiva).

2. Control de los contaminantes producidos en plantas industriales y químicas, así como también en establecimientos de elaboración de alimentos en zonas urbanas (panaderías, pollerías, restaurantes, etc.).

3. Control de contaminación Vehicular mediante las revisiones técnicas periódicas, disminuir los vehículos antiguos y en malas condiciones de funcionamiento.

\section{REFERENCIAS BIBLIOGRÁFICAS}

[1] ARAGÓN A., CAMPOS A., LEYVA R., HERNÁNDEZ M., BONITO L.A. Modelos para el cálculo de monóxido de carbono en avenidas (1992). En: Contaminación del aire y salud. Serie Salud Ambiental, No. 2. La Habana. Instituto Nacional de Higiene, Epidemiología y Microbiología. México D.F. Ciencias Médicas. 1992:79-91.

[2] CHARPENTIER, S. y HIDALGO, J. (2000). Políticas Ambientales en el Perú, Ed. Agenda Perú.

[3] FUNDACIÓN MAPFRE (1998).Planificación y Control de la Contaminación Ambiental, M. Soares Calvo, Itsemap. Madrid

[4] GIRALDO, A. y LOAYZA, S. (2001). UNMSM. Facultad de Ingeniería Geográfica. El índice de calidad ambiental y calidad de vida. Ministerio de Salud. http://www.minsa.gob.pe. [Visitado: 04-11-2010].

[5] KIELY, G. (1999). Ingeniería Ambiental. Fundamentos, Entornos, Tecnologías y Sistemas de Gestión. Madrid. McGraw-Hill/Interamericana de España S.A.U. Volumen II, Páginas 458 a 460.

[6] Medio Ambiente en el Perú (2000), Instituto Cuanto USAID.

[7] PROGRAMA NACIONAL DE VIGILANCIA SANITARIA DE CALIDAD DEL AIRE (2010). En: $<$ http://www.digesa.minsa.gob.pe/DEPA/caire/ lima_callao.asp>. [Visitado: 03-11-2010].

[8] RIVERA POMA, Juan Manuel (2011) Tesis de Grado: "Modelo de Identificación de Factores Contaminantes Atmosféricos críticos en Lima Callao"

[9] ROMERO Placeres M, MÁS Bermejo P, LACASAÑA NAVARRO M, TÉLLEZ ROJO Solís M, AGUILAR VALDÉS J, Romieu I. (2004). Contaminación atmosférica, asma bronquial e infecciones respiratorias agudas en menores de edad de La Habana. Sal. Pública México. 46:222-3. 